\title{
EDUCACIÓn PARA EL DESARROLLO SOSTENIBLE [EDS] Y ARQUITECTURA ESCOLAR. EL ESPACIO COMO REACTIUO DEL MODELO PEDAGÓGICO
}

\section{The relationship between architecture and Education for Sustainable Development (ESD]. Space as a reactive factor within the pedagogical model}

\author{
BEATRIZ AMANN VARGAS \\ Istituto Europeo di Design Madrid
}

DOI: 10.13042/Bordon.2016.68109

Fecha de recepción: 20/06/2015 - Fecha de aceptación: 22/10/2015

Autora de contacto / Corresponding Author: Beatriz Amann Vargas. Email: b.amann@madrid.ied.es

INTRODUCCIÓN. La Educación para el Desarrollo Sostenible (EDS) promueve modelos pedagógicos participativos, en los que se fomente la motivación del alumnado y su autonomía a través de la adquisición de pensamiento crítico fundamentado en los valores de sostenibilidad. Todo ello significa, entre otras modificaciones de base, la introducción de cambios en los métodos pedagógicos actuales. ¿Significa también la necesidad de una arquitectura escolar diferente?, ¿puede el espacio arquitectónico acompañar positivamente la EDS? El objetivo que guía este trabajo es identificar posibles criterios de diseño para un espacio reactivo del modelo pedagógico coherente con la EDS. MÉTODO. El estudio utiliza una metodología inductiva que trabaja con una herramienta concreta de análisis, la crítica poética de arquitectura, que analiza las interacciones entre los sistemas que conforman un espacio. De este modo, pueden identificarse relaciones de convergencia y divergencia entre modelos educativos y cualidad espacial. Se toman como referencia tres centros escolares internacionales que implementan modelos pedagógicos incluyentes de los valores y criterios de la EDS. Estos centros han sido construidos durante la década 2005-2014, periodo que marca los objetivos de la UNESCO para el desarrollo de la EDS. RESULTADOS. El artículo defiende que existe una interacción entre modelo pedagógico y espacio educativo. Como hallazgo principal, identifica diez criterios de diseño particulares y dos más generales para que el espacio escolar sea un espacio acompañante de modelos pedagógicos afines a la EDS. DISCUSIÓN. Por un lado, los resultados muestran afinidad con las teorías de la neuroeducación, como ciencia emergente. Por otro lado, el estudio encuentra su límite, precisamente, en el logro de sus objetivos; ya que los criterios identificados son postulados abiertos. Estos resultados apuntan a la necesidad de renovar la normativa estatal existente respecto a la arquitectura de centros escolares públicos de manera que se posibilite la implantación de modelos pedagógicos innovadores.

Palabras clave: Arquitectura escolar, Entorno físico, Espacio escolar, Criterios de diseño, Educación abierta. 


\section{Un desarrollo sostenible}

Hace ahora casi tres décadas, la propuesta inicial de la Teoría del Desarrollo a Escala Humana (Max Neef, Elizalde, y Hopenhayn, 1986; Max Neef, 2006) afirmó que las necesidades fundamentales de una persona son invariables y finitas, en toda época y en cualquier lugar. Esta teoría enuncia cuatro necesidades de tipo ontológico (ser, estar, tener y hacer) y nueve más de tipo axiológico (protección, afecto, entendimiento, participación, ocio, creación, identidad, libertad y subsistencia) como aquellas necesidades potenciales para mejorar las condiciones de vida de los seres humanos. Lo que varía, a lo largo del tiempo y en los diferentes sistemas culturales, políticos y económicos, es la manera en que estas se satisfacen: los medios "satisfactores". Así, se propone una evolución social y económica en base a las necesidades de las personas.

Un año después, al amparo de la Organización de Naciones Unidas, el Informe de la Comisión Bruntland (1987) describe el concepto de desarrollo sostenible como un "desarrollo que satisface las necesidades de la generación presente, sin comprometer la capacidad de las generaciones futuras de satisfacer sus propias necesidades". Así, en este informe, se añade al concepto de necesidad fundamental el de desarrollo sostenible, para el que se describen cuatro dimensiones: la sociedad, la cultura, la economía y el medio ambiente. Estos sistemas no son independientes entre sí, sino que, tal y como ya afirmaba Morin (1980), se da una ecoorganización de sinergias entre ellos. De este modo, se presenta la sostenibilidad como la característica de equilibrio necesaria entre el medio ambiental, económico y social, en pos de una mejora global en la calidad de vida. En este sentido, el concepto de sostenibilidad se refiere tanto a la calidad ambiental como a la justicia social y a la economía equitativa (Aznar y Ulls, 2009). Y el desarrollo sostenible alude a los procesos para conseguir este objetivo. De este modo, el desarrollo sostenible extiende los valores de un desarrollo a escala humana desde el presente hasta las generaciones venideras, de modo que aúna las necesidades de la especie humana con las necesidades para la conservación de la biodiversidad.

\section{Una educación para el desarrollo sostenible}

En el año 2002, la Asamblea General de las Naciones Unidas promulga la Resolución $n^{\circ}$ 57/254 bajo el título "Decenio de las Naciones Unidas de la Educación para el Desarrollo Sostenible 2005-2014", inspirada, entre otros, en La Carta de la Tierra (2000). En ella, para alcanzar los valores de sostenibilidad en la sociedad presente y futura, se presenta como fundamental el papel de la educación. Durante esta década, la UNESCO trata de implementar esta acción con el fin de lograr sus objetivos: alcanzar una educación para el desarrollo sostenible (EDS) globalizada ${ }^{1}$. Esta es una propuesta educativa que busca capacitar a la persona en las competencias básicas cognitivas, metodológicas y, también, actitudinales, de cara a interiorizar valores de sostenibilidad y principios epistemológicos. Se intenta incorporar así, a los currículos escolares y universitarios, criterios de compromiso y toma de conciencia con temas como el cambio climático, la biodiversidad o el consumo sostenible (Aznar y Ulls, 2009). Al mismo tiempo, la EDS promueve modelos pedagógicos participativos, en los que se fomente la motivación del alumnado y la adquisición de pensamiento crítico.

Estos objetivos se vuelven a reforzar en la nueva hoja de ruta que elabora la UNESCO en el año 2014, una vez finalizado el Decenio, "UNESCO Education Strategy 2014-2021", con el fin de dar continuidad a los logros alcanzados y para promover acciones que refuercen la creatividad y la ciudadanía responsable entre los estudiantes (UNESCO, 2014). Así, se trata de mejorar la calidad de la enseñanza y de respetar la diversidad ${ }^{2}$. Por un lado, a través de la 
innovación pedagógica y curricular. Por otro, a través de la renovación de los sistemas educativos que refuercen a los docentes en el uso de metodologías interactivas y de desarrollo de capacidades. Por lo tanto, se subraya la necesidad de fomentar pedagogías respetuosas con las capacidades y con el potencial del individuo "atendiendo a las diferentes necesidades y habilidades cognitivas y emocionales".

Esto requiere una transformación integral del sistema educativo actual-tradicional mayoritariamente extendido (Larrañaga, 2012). Revisión que incluye, también, la adaptación de los centros escolares, de su cualidad espacial - cuestión en la que se centra esta investigación-, para poder permitir la innovación práctica descrita, para ser respetuosos con la diversidad y para facilitar las nuevas posibilidades de comunicación, aprendizaje y de compartir el conocimiento que proporcionan las $\mathrm{TIC}^{3}$.

\section{La consideración del espacio en el hecho educativo}

Atendiendo, de nuevo, a la Teoría del Desarrollo a Escala Humana (Max Neef et al., 1986; Max Neef, 2006), el hecho arquitectónico participa como medio satisfactor de alguna de las necesidades fundamentales del individuo. En esta teoría se alude a la morada "la vivienda" como satisfactor de la necesidad combinada protección-estar; los espacios de encuentro satisfacen parte de la necesidad combinada afecto-estar; los espacios públicos de esparcimiento, los ambientes y paisajes, son satisfactores de la necesidad combinada ocio-estar; y los ámbitos de interacción formativa "los que más interesan a este trabajo, como escuelas, colegios y universidades" colmatan la necesidad combinada entendimiento-estar.

Esto exige considerar el espacio arquitectónico en los procesos sociales como algo fundamental. La sociología del espacio, desarrollada sobre todo en el ámbito de lo urbano (Sennett,
1996; Leal, 1997; Gehl, 2011), establece que cualquier reestructuración social importante conlleva una revisión de la cualidad espacial.

La sociología de los atributos espaciales, como prefiere denominarla Leal (1997), se construye, así, a través de tres perspectivas complementarias: 1) La que establece cómo se concibe el espacio en relación con los fenómenos sociales; 2) la que identifica los procesos de la acción social en relación con la cualidad espacial, pero siendo consciente de su limitación; y 3) la que percibe la relación entre los elementos sociales estructurales y el espacio como una relación de la actuación del capital.

En todo caso, la sociología de los atributos espaciales asume que el espacio organiza nuestras percepciones de los fenómenos sociales y que tiene carácter estructurador. No obstante, a pesar de los diferentes matices que los distintos autores desarrollan en este campo (Castells, 1997, 2009; Giddens, 1991; Gottdiener, 1994; Harvey, 1989; Kern, 1983; Leal, 1997; Lefebvre, 1976; Sennett, 1996; Simmel, 1924), desde la sociología no se concibe el espacio como algo físico, sino que tiene una concepción relacional de interacción social. Esto es, no se acepta el espacio como una realidad absoluta, sino en su relación con la dimensión temporal.

Perkins (1957) observó que el arquitecto que proyecta un centro escolar no puede pensar solamente en términos de refugio, o de planos, o de ladrillos y piedra y acero. Debe pensar en los futuros usuarios del edificio. Debe pensar en el trabajo que el edificio debería ayudar a acometer: el total desarrollo de los potenciales de cada uno de los alumnos y alumnas del centro. Si el arquitecto no pierde de vista este principio, podrá ser capaz de contribuir al logro de los objetivos de los educadores creando un edificio que es una herramienta para el docente y una expresión del enfoque educativo de la escuela. Creando un ambiente, un estado de ánimo, que ayude al estudiante en cada tarea de aprendizaje. Haciendo que el centro escolar sea 
un lugar al que el alumno desee entrar, un lugar del que no quiera irse.

Los estudios recientes sobre neuroeducación (Mora, 2010), que retoman el enfoque holístico de la psicología del espacio (Moles y Rohmer, 1998), establecen una relación directa entre la cualidad espacial, propiamente dicha "topológica, funcional, material, morfológica ", y el aprendizaje. Es posible pensar, entonces, que el espacio arquitectónico, como realidad física, puede facilitar la EDS. Esta premisa plantea, desde el punto de vista de la cualidad arquitectónica, la relación entre espacio educativo, espacio escolar y acción pedagógica. Así, se intentan matizar los planteamientos de la sociología de los atributos espaciales y de la psicología del espacio con el objetivo de detectar criterios de diseño para el espacio escolar que puedan propiciar el desarrollo de la EDS. Es decir, el objetivo principal que guía este trabajo es identificar posibles criterios de diseño para un espacio arquitectónico reactivo de un modelo pedagógico que avanza en la EDS ${ }^{4}$.

\section{Método}

La investigación que se presenta utiliza una metodología inductiva que trabaja con una herramienta concreta de análisis: el método Mirregan-Todorov para la crítica de arquitectura (Miranda, 1999).

Para profundizar en la cualidad arquitectónica de la arquitectura escolar, este trabajo emplea el cuarto estadio de un método de crítica de arquitectura probado y reconocido (Amann, 2015) como es el método Mirregan-Todorov (Miranda, 1999). Esta fase se conoce como crítica poética y lleva consigo implícitas las fases anteriores de descripción, análisis e interpretación.

La crítica poética es el orden idóneo para estudiar la temática presentada porque se trata de un método que salvaguarda la complejidad del objeto "cuestión fundamental en este caso, que exige analizar e interpretar la interacción entre la realidad espacial y el individuo".

Un objeto arquitectónico está formado por grandes sistemas, entre los que destacan cinco necesarios y suficientes: topología o geometría, programa o función, materia o construcción, morfología o forma, y campo o medio. Para este estudio, y dada su relevancia al respecto, se ha tomado como sistema principal también el subsistema campo educativo. Estos sistemas, cada uno de ellos muy complejo, no son autónomos, sino que interactúan entre sí. Son estas sinergias y fricciones entre sistemas las que determinan el carácter de un objeto arquitectónico (Amann, 2015), de un centro escolar, en este caso.

La crítica poética profundiza en estas interacciones extrapolando comparaciones binarias entre sistemas, para volver, a continuación, a la visión compleja de la totalidad. Esta metodología se ha aplicado, en primer lugar, sobre cada ejemplo y, en un segundo orden, comparando los resultados de los ejemplos entre sí.

\section{Selección de interacciones}

Una crítica poética completa daría lugar a treinta interacciones fruto de la combinación de los sistemas arquitectónicos principales entre sí. Para este estudio, solamente se ha profundizado en aquellas que dan respuesta a los objetivos de la investigación. De esta manera, interesa el análisis de:

- El sistema topología: las dimensiones del espacio (según el número de alumnos, si se permite el movimiento, si es un espacio versátil, si permite el aislamiento, si permite distintos ambientes, su volumen ) y la disposición de elementos en él (si son muchos o pocos, si su posición es ordenada o aleatoria, si son fijos o móviles, si pueden transformarse o no, si son manipulables o no, si se disponen marcando ejes, qué ejes, cuál es 
la relación entre la posición del docente y los alumnos, la relación de la posición de los alumnos entre sí, la posición de la puerta en relación a los elementos ), la escala de los elementos, etc.

- El sistema programa: el uso del espacio (si se utiliza para una comunicación unidireccional, bidireccional o multidireccional, si es participativo, colectivo, si se potencia el aprendizaje autónomo, si se utilizan TIC...), los flujos funcionales (si son dinámicos o estáticos, mixtos, si el espacio es versátil o no, si las circulaciones responden a esquemas rutinarios o no), con qué elementos se cuenta (mesas, sillas, armarios, elementos lúdicos, rincones, elementos deportivos ) y cómo funcionan (si son estáticos y fijos o dinámicos y maleables, si permiten una evolución, si son manipulables según la edad, si son abiertos, si son adaptables ), etc.

- El sistema materia: la luz (si es natural o artificial, su intensidad, su color, su disposición, ), las condiciones ambientales de salubridad (condiciones de humedad y temperatura, la ventilación natural o artificial ) y los materiales de los acabados (las texturas, si son cálidos, la madera, el metal, el caucho, el corcho, la tela, su procedencia, si son reciclados, si son sostenibles), etc.

- El sistema morfología: la forma del espacio (si tiene ángulos, si es un espacio con curvas, si es abierto o cerrado, regular o irregular, si el suelo tiene desniveles, ) y el color (si el espacio es monocromo o policromo, si predomina el blanco, si se da una gama temática, ), su cualidad estética, etc.

- El sistema campo: el modelo educativo (campo educativo), las condiciones sociales y económicas, la condición territorial, etc.

\section{Muestra de la investigación}

En un intento de objetivar criterios para el diseño arquitectónico de centros educativos que propicien el desarrollo de la EDS, se toman como referencia tres centros escolares internacionales representativos de modelos pedagógicos coherentes con los valores y criterios de la EDS (pedagogías participativas e interactivas, fomento de la motivación, la autonomía, el pensamiento crítico, la creatividad y la ciudadanía responsable en la persona, el respeto por la diversidad, las habilidades emocionales y el potencial cognitivo del individuo, la inclusión de valores de sostenibilidad, el uso de TIC, etc.). Estos centros escolares han sido construidos durante la década 2005-2014, periodo que marca los objetivos de la UNESCO para el desarrollo de la EDS (Organización de Naciones Unidas, 2002).

La selección de casos se ha hecho siguiendo tres criterios que favorecen la metodología empleada: a) casos de centros escolares de etapas distintas; b) casos de centros escolares de latitudes y culturas diferentes; c) casos de centros escolares que trabajan modelos pedagógicos acordes con los valores y criterios de la EDS.

Para la búsqueda de casos, se ha consultado bibliografía específica y actualizada (Casqueiro, 2014). Una vez realizada la selección, se ha contactado directamente con los estudios de arquitectura de los autores de los proyectos elegidos y con los centros escolares para recoger los datos de investigación ${ }^{5}$. Los centros de la muestra son los siguientes ${ }^{6}$ :

- Fuji Kindergarten ${ }^{7}$. Localización: Tachikawa (Japón). Año de apertura: 2007. Etapa educativa: equivalente a educación infantil. Modelo pedagógico: Montessori. Autor: Tezuka Architects.

- Ramat Chen Arts E Science Elementary School. Nuevo hall de acceso a primer grado ${ }^{8}$. Localización: Ramat Gan (Israel). Año de apertura: 2014. Etapa educativa: equivalente a educación primaria. Modelo pedagógico: Sistema Educativo Ramat Gan. Autor: Sarit Shani Hay.

- Vittra School Brotorp9 . Localización: Brotorp (Suecia). Año de apertura: 2012. Etapa educativa: equivalentes a educación infantil, educación primaria y ESO. 
Modelo pedagógico: Sistema Educativo Sueco. Autor: Rosan Bosch.

\section{Resultados}

Los resultados de aplicar la crítica poética a la muestra estudiada se presentan en sendos subapartados atendiendo a los ejes de interacción entre los sistemas previamente establecidos, un total de seis. La interpretación de los resultados permite avanzar en cada caso los criterios de diseño para un espacio reactivo del modelo pedagógico (EDS)

\section{Criterios de diseño para un espacio reactivo del modelo pedagógico (EDS)}
Criterios de diseño a partir del estudio de la interacción entre los sistemas campo educativo-topología

Tabla 1. Muestra de resultados de la interacción entre los sistemas campo educativo y topología

Interacción campo educativo-topología

$\begin{array}{ccc}\begin{array}{c}\text { Flexibilidad de la estructura } \\ \text { espacial }\end{array} & \begin{array}{c}\text { Relación escala elementos } \\ \text { espaciales-usuarios }\end{array} & \begin{array}{c}\text { Superficie de desarrollo del } \\ \text { espacio de aprendizaje }\end{array}\end{array}$
espacial espaciales-usuarios espacio de aprendizaje

\begin{tabular}{|c|c|c|c|}
\hline Caso A & $\begin{array}{l}\text { - Ausencia de particiones: } \\
\text { desaparece el "espacio "aula" } \\
\text { como tal } \\
\text { - Apropiación del espacio } \\
\text { interior y exterior para } \\
\text { actividades pedagógicas } \\
\text { - Existencia generalizada de } \\
\text { espacios de participación } \\
\text { y uso colectivo } \\
\text { - Uso total del espacio: interior, } \\
\text { exterior y cubierta }\end{array}$ & $\begin{array}{l}\text { - Adaptación ergonómica de } \\
\text { elementos a la edad de los } \\
\text { usuarios: } \\
\text { - Dimensión de lavabos e } \\
\text { inodoros } \\
\text { - Dimensión de elementos } \\
\text { móviles para creación de } \\
\text { mobiliario } \\
\text { - Altura de fuente } \\
\text { en el patio }\end{array}$ & $\begin{array}{l}\text { - Al no existir un espacio } \\
\text { cerrado de aula, la superficie } \\
\text { de multiplica: todos los m2 son } \\
\text { útiles pedagógicamente } \\
\text { - Nuevos espacios pedagógicos: } \\
\text { cubierta, lavabos, fuente, límite } \\
\text { interior-exterior }\end{array}$ \\
\hline Caso B & $\begin{array}{l}\text { - Apropiación del espacio } \\
\text { común (acceso) para } \\
\text { actividad pedagógica y } \\
\text { participativa }\end{array}$ & $\begin{array}{l}\text { - Adaptación ergonómica de } \\
\text { elementos a la edad de los } \\
\text { usuarios: } \\
\text { - Barra de danza } \\
\text { - Cubículo audiovisual } \\
\text { - Altura pantalla interactiva } \\
\text { - Rincones de lectura }\end{array}$ & $\begin{array}{l}\text { - El acceso al centro es un } \\
\text { espacio social y de juego } \\
\text { creativo de gran superficie } \\
\text { organizada en cuatro } \\
\text { actividades: } \\
\text { - Gran pizarra magnética } \\
\text { - Actividad psicomotriz } \\
\text { - Actividad auditiva } \\
\text { - Actividad lectora }\end{array}$ \\
\hline aso $C$ & $\begin{array}{l}\text { - Espacios de circulación como } \\
\text { extensión del "espacio aula" } \\
\text { - Organización de estructuras } \\
\text { espaciales en espacios de } \\
\text { circulación para promover } \\
\text { la actividad pedagógica } \\
\text { participativa }\end{array}$ & $\begin{array}{l}\text { - Adaptación ergonómica de } \\
\text { elementos a la edad de los } \\
\text { usuarios: } \\
\text { - Escala del mobiliario por } \\
\text { zonas (infantil y primaria) }\end{array}$ & $\begin{array}{l}\text { - Combinación de rincones } \\
\text { de transición entre aulas y } \\
\text { espacio común: espacios de } \\
\text { aprendizaje individual } \\
\text { - Multiplicación de tipología } \\
\text { espacial al coexistir el "espacio } \\
\text { aula" con los nuevos espacios } \\
\text { propuestos (en circulaciones) } \\
\text { - Distinta tipología y dimensión } \\
\text { de aulas según actividad } \\
\text { buscada: individual o } \\
\text { participativa }\end{array}$ \\
\hline
\end{tabular}

Fuente: elaboración propia. 
Todos los espacios son espacios potenciales para el aprendizaje

Y en este sentido se proyectan. Las estructuras espaciales son lo más flexibles posible. Tanto en lo que se refiere a espacios interiores, como exteriores. Porque todo el espacio se considera como espacio útil para la pedagogía. Esto es, no solo se aprende dentro del aula.

Este hecho aparece con fuerza en los espacios de circulación, que pasan a convertirse en una extensión del aula, como veremos más adelante al hablar de la Learning-street (Hertzberger, 2008).

La escala de los elementos es acorde a la escala del niño

Se perciben cambios en el diseño ligados a los modelos pedagógicos EDS. Así, tanto las proporciones del espacio como la escala y ergonomía del mobiliario se adaptan a la edad de los usuarios en un diseño inclusivo. Las dimensiones y las alturas de ventanas, puertas, lavabos, espejos, estanterías, interruptores potencian la iniciativa, la participación y la autonomía de los alumnos y alumnas.
Necesidad de una superficie mayor de desarrollo

El fomento del aprendizaje a través del juego creativo, del contacto social y de la expresión natural precisa de un espacio amplio, que permita el movimiento. Se trata de propiciar un catálogo más amplio de lugares y rincones —algunos para las actividades colectivas y otros en los que sea posible la concentración y el aprendizaje individual, sin ser molestado-.

Figura 2. Espacio para la concentración y el trabajo individual en la Vittra School Brotorp

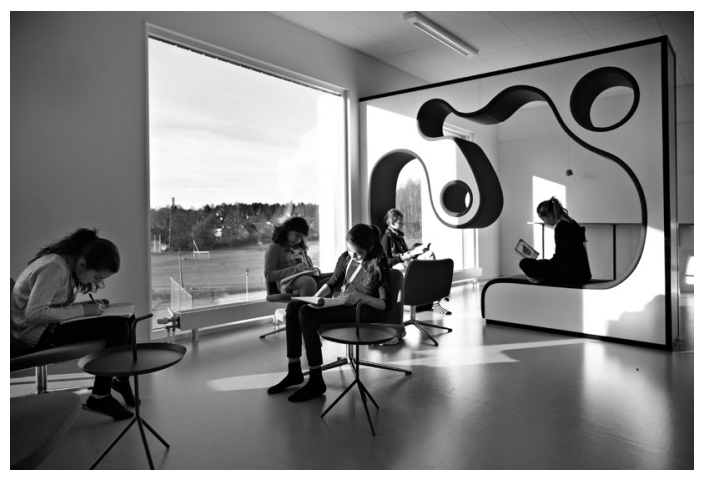

Fotografía de Kim Wendt, estudio Rosan Bosch.

Figura 1. Planta de la Vittra School Brotorp, estudio Rosan Bosch

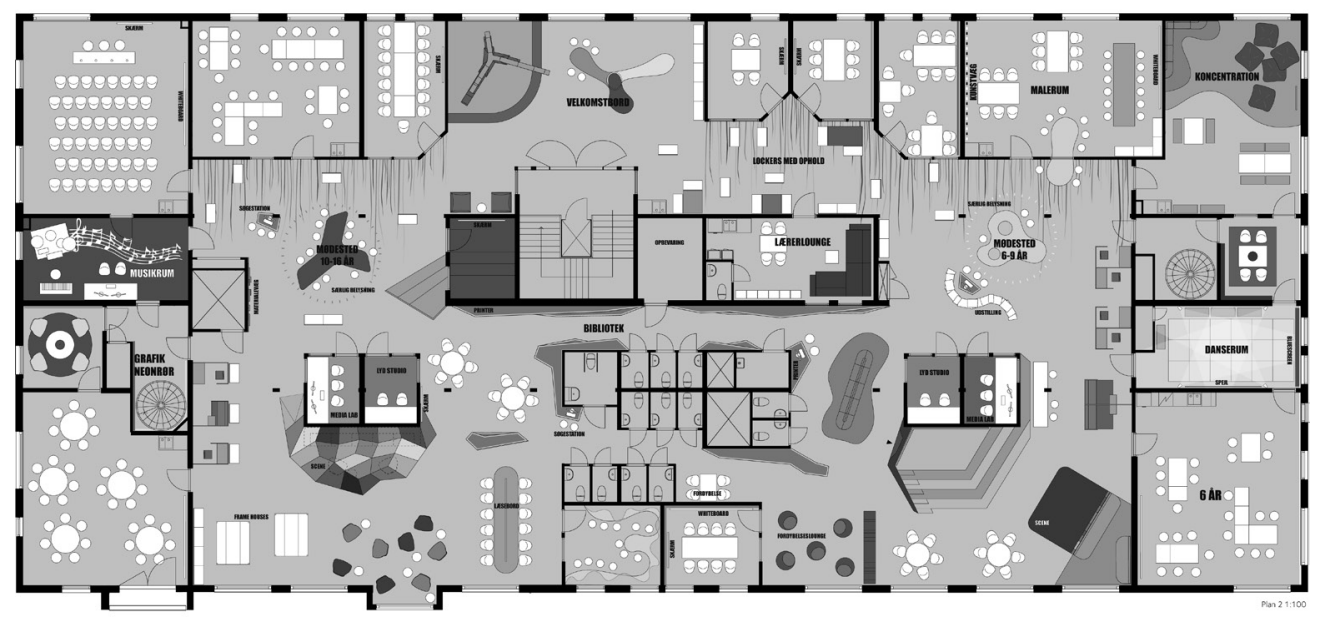

Nota: Obsérvese el tratamiento y la estructuración de los espacios de circulación como espacio útil para el aprendizaje. 
De esta manera, se detecta que, para hacer posible la inclusión de usos colectivos y espacios de trabajo en grupo en el aula, hay que incrementar la dimensión de la misma. Los modelos educativos acordes con la EDS promueven el trabajo colaborativo - tanto en grandes como en pequeños grupos- para el desarrollo cognitivo. Aparece, por tanto, la necesidad de diseñar espacios que puedan acoger actividades tales como el diálogo y la crítica, la negociación, el planteamiento y la resolución de problemas, etc.

Figura 3. Entorno flexible para la interacción y la participación en la Vittra School Brotorp

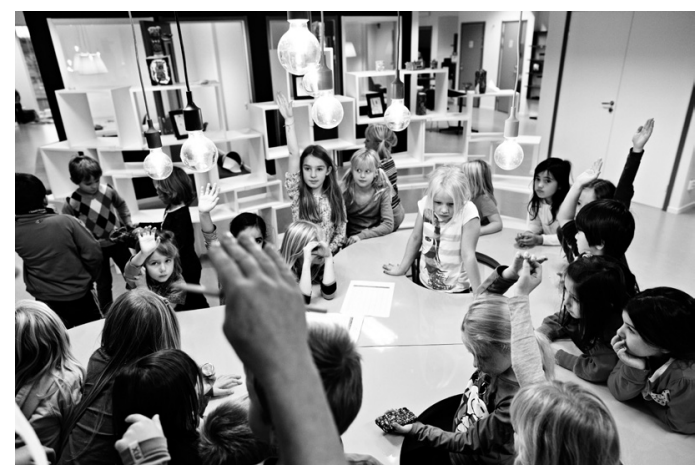

Fotografía de Kim Wendt, estudio Rosan Bosch.

Criterios de diseño a partir del estudio de la interacción entre los sistemas campo educativo-programa

El programa se adapta al modelo educativo y a las necesidades específicas del contexto

Lo que significa que es, precisamente, en el reparto interno de los usos donde se detecta la interacción entre un sistema pedagógico concreto y la cualidad espacial de la arquitectura escolar. En los casos de estudio analizados, la diversidad espacial hace referencia a programas educativos complejos, en los que se trabajan formas de aprendizaje diversas. En este sentido, la interacción entre campo educativo y programa espacial es indispensable.
Tabla 2. Muestra de resultados de la interacción entre los sistemas campo educativo y programa

Interacción campo educativo-programa

Adaptación de usos

Caso A Escuela infantil con modelo pedagógico participativo Montessori:

- Ausencia de mobiliario fijo

- Elementos móviles que posibilitan el desarrollo de programas y usos diversos - Accesibilidad universal

Caso B Propuesta espacial adaptada al Learning by Playing y al carácter del centro enfocado a cinco áreas:

- Artes gráficas

- Teatro

- Música

- Danza

- Ciencia

Caso C La adaptación programática se da a través de la diversidad espacial:

- Espacio aula mesas colectivas

- Espacio aprendizaje individual

- Espacio claustro

- Espacio debate

- Espacio proyectos

- Espacio multiusos

Fuente: elaboración propia.

Figura 4. La planta del Fuji Kindergarten

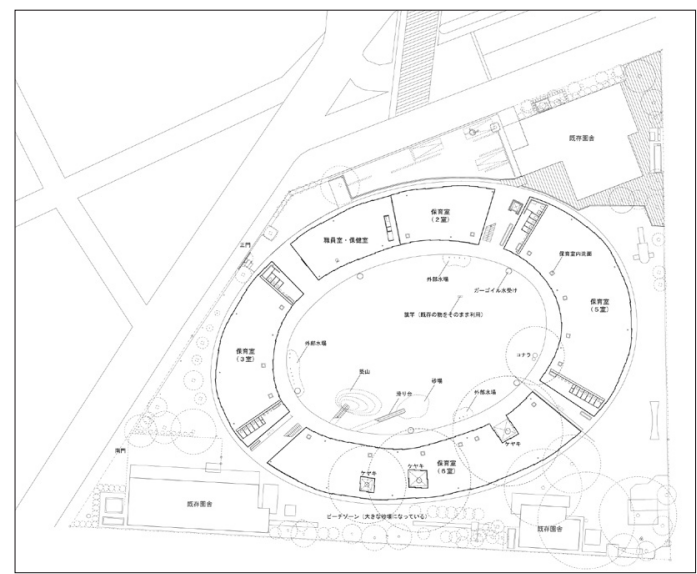

Estudio Tezuka Architects, sin mobiliario ni particiones físicas entre los espacios interiores, presenta un modelo educativo que hace hincapié en la estimulación emocional y cognitiva del niño a través de proyectos abiertos. 


\section{Criterios de diseño a partir del estudio de la interacción entre los sistemas campo educativo-materia}

Tabla 3. Muestra de resultados de la interacción entre los sistemas campo educativo y materia

Interacción campo educativo-materia

$\begin{array}{llc}\text { La materia como estímulo } & \text { La materia como acción } & \begin{array}{c}\text { Desarrollo tecnológico y } \\ \text { sostenibilidad }\end{array}\end{array}$

Caso A - La ausencia de materialidad en la fachada llena los espacios de luz natural

- Uso global de madera natural en los elementos, que otorga calidez al espacio

- La transparencia de los espacios como estímulo del bienestar y lo dinámico

- El uso simbólico del color como signo

\begin{tabular}{|c|c|c|c|}
\hline Caso B & $\begin{array}{l}\text { - La materialización propicia la } \\
\text { estimulación de los sentidos y el } \\
\text { sentimiento de pertenencia: } \\
\text { - El uso simbólico del color como } \\
\text { signo; el uso de la psicología del } \\
\text { color (suelo de cuadrícula blanca- } \\
\text { negra que invita al movimiento } \\
\text { físico; rincones de lectura en } \\
\text { verde para un efecto relajante) } \\
\text { - Diversidad de elementos } \\
\text { en función de la actividad } \\
\text { propuesta (rincones de lectura } \\
\text { y socialización que combinan } \\
\text { mesas, estanterías, cajones, etc.) } \\
\text { - Espacio bien iluminado: espacio } \\
\text { dinámico } \\
\text { - Utilización de elementos } \\
\text { fonoabsorbentes para posibilitar la } \\
\text { diversidad de usos }\end{array}$ & $\begin{array}{l}\text { - Un único espacio utiliza diferentes } \\
\text { materiales y colores para } \\
\text { caracterizar actividades diversas: } \\
\text { - Superficie de cuadrícula para } \\
\text { el movimiento ante pantalla } \\
\text { interactiva } \\
\text { - Rincones de lectura y socialización } \\
\text { que combinan estructura de } \\
\text { madera y el confort del textil en } \\
\text { los asientos. La dimensión del } \\
\text { espacio crea intimidad y favorece la } \\
\text { socialización } \\
\text { - Rincón audiovisual con material } \\
\text { fonoabsorbente } \\
\text { - Paño magnético para decoración } \\
\text { mural colectiva }\end{array}$ & $\begin{array}{l}\text { - Uso de TIC: } \\
\text { - Pantalla interactiva de } \\
\text { gran formato } \\
\text { - Rincón de audio (gran } \\
\text { pantalla dotada de } \\
\text { tecnología audiovisual } \\
\text { que permite el juego } \\
\text { interactivo, el juego } \\
\text { auditivo y el visionado } \\
\text { de películas) }\end{array}$ \\
\hline Caso C & $\begin{array}{l}\text { - Buena iluminación natural en las } \\
\text { aulas } \\
\text { - Uso del color en los elementos } \\
\text { espaciales propuestos en la } \\
\text { circulación: uso simbólico } \\
\text { del color } \\
\text { - Propuesta de diferentes texturas } \\
\text { para actividades diversas: } \\
\text { proyectos, lectura, juego: de la } \\
\text { madera al textil }\end{array}$ & $\begin{array}{l}\text { - Para la diversificación de usos se } \\
\text { combinan tres elementos: } \\
\text { - Madera (al natural o lacada en } \\
\text { color): mobiliario para proyectos y } \\
\text { debate } \\
\text { - Textil: moqueta: en zonas } \\
\text { estanciales (grada) } \\
\text { - Textil: tapizado de mobiliario: en } \\
\text { espacios de estudio individual } \\
\text { - Topografía artificial en espacios } \\
\text { de circulación: desniveles para } \\
\text { incentivar el movimiento }\end{array}$ & $\begin{array}{l}\text { - Uso de TIC: } \\
\text { - Uso generalizado de } \\
\text { tablets y portátiles, } \\
\text { también fuera del } \\
\text { aula (espacios de } \\
\text { investigación personal) }\end{array}$ \\
\hline
\end{tabular}

Fuente: elaboración propia.
- Cuerdas y líneas de flujos en el espacio exterior: incitación al movimiento

- Arenero: incitación al juego experimental

- Árbol: incitación al desarrollo de la imaginación

- Suelos duros y blandos
- Criterios sostenibles de diseño:

- Respeto de especies arbóreas existentes antes de la construcción

- Uso consciente del agua

- Huerto colectivo
Un único espacio utiliza diferentes materiales y colores para caracterizar actividades diversas: interactiva

ncones de lectura y socialización que combinan estructura de espacio crea intimidad y favorece la socialización

Rincón audiovisu

Paño magnético para decoración mural colectiva
- Uso de TIC:

gran formato

Rincón de audio (gran pantalla dotada de auditivo y el visionado de películas) 
Las características físicas de los materiales como elemento de estimulación

La luz, el color, las texturas, el sonido, el olor, la temperatura pueden conjugarse con el fin de lograr un microclima propicio para el aprendizaje: estimulante, sorprendente, confortable y familiar. Se puede afirmar que el ambiente del centro escolar puede sumarse como herramienta pedagógica, adaptándose tanto al modelo pedagógico como a cada figura docente.

Así, por ejemplo, se busca el uso de la luz natural, siempre que sea posible. Utilizando, además, cambios en la iluminación para señalar espacios con actividad diferente. Por otro lado, se emplean materiales fonoabsorbentes para minimizar o adecuar el impacto del ruido.

Las características físicas de los materiales se utilizan como elemento de acción

FIGURA 5. Suelo lúdico y pared con imán. Ramat Chen Arts \& Science Elementary School, nuevo hall de entrada, estudio Sarit Shani Hay

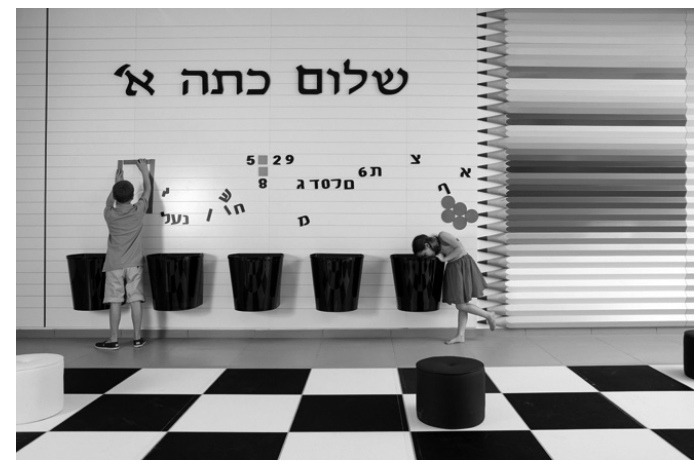

Fotografía de Shiran Carmel.

Los modelos educativos que representan los valores y criterios de la EDS trabajan con múltiples formas de exploración y representación. Estas pedagogías integran las artes gráficas como instrumento para el desarrollo cognitivo, lingüístico y social. Para posibilitar esta forma activa de aprendizaje, el espacio "su mobiliario, sus equipamientos, etc." ha de ser capaz de acoger actividades tales como el teatro y la música, la pintura, la construcción, el deporte, etc. Y este condicionante incide directamente en la necesidad de diseñar entornos con superficies versátiles y especializadas.

También en el área exterior, en la que conviven texturas diversas y condiciones ambientales estudiadas: arena, hierba, topografías artificiales, suelos duros y blandos, sombras, espacio abierto, etc.

Las estrategias de diseño se ligan al desarrollo tecnológico y a la sostenibilidad

Por un lado, el acompañamiento de las TIC se incorpora a las formas de aprendizaje.

Por otro lado, se promueve la utilización de materiales con un recorrido sostenible, tanto en el proceso de fabricación y montaje como en su vida útil y su versatilidad en el uso.

FIgURA 6. Rincón musical. Ramat Chen Arts $\&$ Science Elementary School, nuevo hall de entrada, estudio Sarit Shani Hay

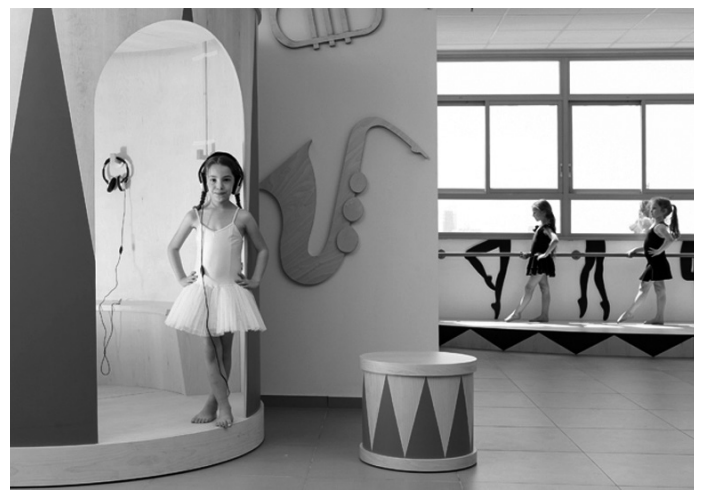

Fotografía de Shiran Carmel. 


\section{Criterios de diseño a partir del estudio de la interacción entre los sistemas campo educativo-morfología}

TABla 4. Muestra de resultados de la interacción entre los sistemas campo educativo y morfología

Interacción campo educativo-morfología

La morfología como paisaje de aprendizaje

Caso A - La ausencia de elementos separadores (1. Entre espacios interiores; 2. Entre interior y exterior) promueve conexiones a todos los niveles

- El plano de la cubierta delimita la morfología y se convierte en paisaje

- La morfología del centro (oval) propicia el flujo de actividad, la circulación y lo dinámico

- La ausencia de esquinas diluye el espacio

- La presencia del gran árbol es elemento protagonista, ante la ausencia de otras referencias.

Caso B - El espacio interior crea cuatro paisajes diferenciados a partir de morfologías bien definidas:

- Rincones de lectura: cubículos de madera tipo vagón

- Rincón audiovisual: cubículo cilíndrico que reproduce un tambor

- Rincón teatro: escenario

- Actividad física: superficie plana en el suelo bien definida

Caso C - El espacio interior de circulación se diseña como un paisaje de topología diversa. No solo en lo material, sino en lo volumétrico, desniveles, perspectivas, etc.

Fuente: elaboración propia.

Figura 7. Sección del Fuji Kindergarten, estudio Tezuka Architects

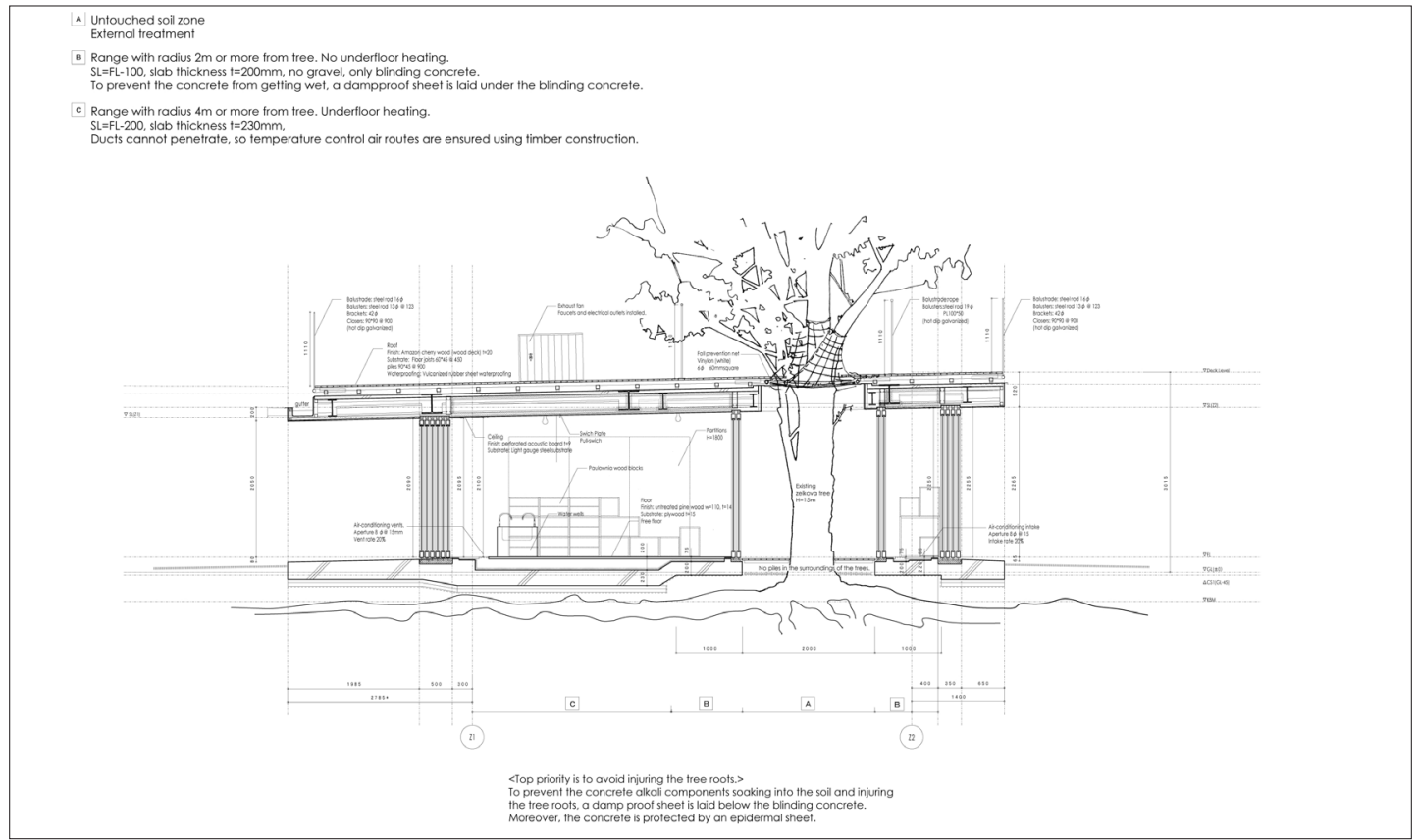

La presencia del árbol se convierte en elemento protagonista de interacción con los usuarios, tanto en el interior como en el exterior (espacio de cubierta usable). 
La forma arquitectónica del centro escolar se convierte en un paisaje para el aprendizaje

Como ya se ha citado, las pedagogías acordes con el modelo EDS precisan de nuevos espacios de aprendizaje que el modelo educativo actualtradicional no contempla. Los nuevos modelos, más avanzados, buscan crear el espacio de aprendizaje total. Para ello, tanto el continente como el contenido de la arquitectura escolar se convierten en protagonistas de este cambio. El centro educativo es un paisaje para el aprendizaje. Cada elemento formaliza un estímulo pedagógico. Para ello, en lo que a la arquitectura concierne, se diluyen los elementos de límite espacial. Esto es, se crean fuertes conexiones entre el interior y el exterior de las aulas; a veces, incluso, los límites entre aula y espacio de circulación desaparecen o se convierten en espacios de transición que acogen otras actividades.

\section{Criterios de diseño a partir del estudio de la interacción entre los sistemas topología-programa}

Las nuevas formas de aprendizaje llevan a modificar el uso de algunos espacios y elementos

Este hecho viene caracterizado, principalmente, por la conversión del espacio de circulación o pasillo en un espacio de relación o Learningstreet (Hertzberger, 2008), en un espacio para el trabajo - tanto colectivo como individual-. De este modo, el aprendizaje continúa fuera del aula.

Para posibilitar esta acción, el diseño del espacio común de circulación toma gran importancia en los proyectos de arquitecturas escolares que desarrollan un modelo pedagógico acorde con los valores y criterios de la EDS. Por una parte, estos espacios adquieren mayor dimensión. Por otra parte, se trabaja su topología al detalle. Ya no tiene por qué tratarse de una estructura linear, sino que puede desarrollar otras geometrías más estimulantes. Además, se incluyen elementos tanto materiales como de mobiliario que fomentan las actividades propuestas en cada caso.

Tabla 5. Muestra de resultados de la interacción entre los sistemas topología y programa

Interacción topología - programa

Nuevos usos espaciales

Caso A - La ausencia de elementos separadores hace de todo el espacio un espacio para el aprendizaje

- No existe espacio jerarquizado

Caso B - El acceso deja de ser un espacio institucional y de representación para ser un espacio lúdico y de aprendizaje colectivo

Caso C - Aprovechamiento del espacio de circulación como espacio de relación o Learning-street: espacio para el aprendizaje y el trabajo colectivo e individual.

- Espacio de circulación de dimensión generosa

- Diseño de elementos específicos para el espacio de circulación: nuevas topologías (ej. escalones o gradas, mobiliario especial)

- Conversión del elemento lineal de circulación en un recorrido de actividad y relación social

Fuente: elaboración propia.

No obstante, la Learning-street no es la única estructura espacial que modifica su uso. En segundo lugar, a veces interrumpiendo, precisamente, la continuidad del espacio de relación, se encuentran los escalones como espacio de transición — diferentes de las escaleras que unen los distintos pisos del edificio-. Este elemento topológico no es nuevo, pero sí su función. Las arquitecturas escolares recientes utilizan estos desniveles como lugar de encuentro entre iguales. Para estimular que los niños y niñas entiendan este espacio como elemento de estancia, y no solo como estructura de paso, se emplean diferentes estrategias: dimensiones generosas, geometrías diversas, y materiales cálidos como la madera o el textil, que ayuden a crear un ambiente de confort. 
Figura 8. Escalones en la Vittra School Brotorp, estudio Rosan Bosch

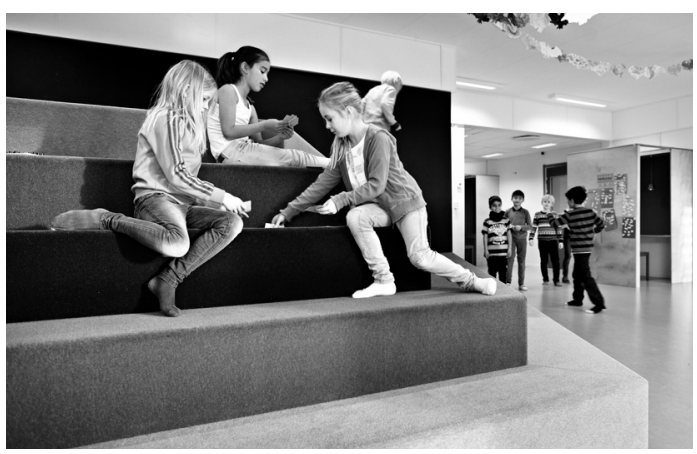

Fotografía de Kim Wendt.

Criterios de diseño a partir del estudio de la interacción entre los sistemas campo-programa

El entendimiento del centro escolar como un equipamiento sociocultural

Este criterio mantiene una estrecha relación con las condiciones socioculturales y económicas del lugar y no se corresponde con ninguno de los casos estudiados. No obstante, se piensa que su inclusión en este listado es fundamental por su estrecha correspondencia con el desarrollo de los valores y los criterios de la EDS.

Este criterio propone entender el centro escolar como un equipamiento que da servicio no solo a los niños y niñas que acuden a él como alumnos y alumnas, sino también a la comunidad en la que el centro se localiza. La extensión del espacio educativo.

Este hecho de incorporar equipamientos culturales del barrio - como pueden ser bibliotecas, espacios deportivos, etc.- puede estimular, además de las capacidades y valores propios de su uso específico, la integración, la inclusión y el respeto por la diversidad que la EDS promulga. En este sentido, este criterio de diseño se presenta como una condición deseable en las arquitecturas escolares acompañantes de la EDS.
Criterios de diseño generales desde la visión compleja

En los puntos anteriores, se enumeran criterios posibles para el diseño de espacios escolares reactivos de modelos educativos afines a los valores de la EDS. Estos criterios se infieren del estudio de las sinergias entre parejas de sistemas que conforman una arquitectura escolar.

A continuación, se vuelve a la visión compleja de la totalidad para destacar dos criterios esenciales "que implican una interacción de todos los sistemas señalados y, por tanto, se alimentan, además, de los criterios ya expuestos, que los engloban ${ }^{10 "}$ que se revelan como condiciones principales de los espacios arquitectónicos educativos afines a la EDS estudiados.

El centro escolar se estructura a partir de espacios multifuncionales

El espacio para la EDS se proyecta con dimensiones y proporciones versátiles; esto es, que sean aptas para la adaptación durante su funcionamiento a las actividades previstas en cada momento. Para lograrlo, el diseño de la planta es flexible y abierto, y se utiliza material polivalente para su construcción "revestimientos y superficies, elementos de mobiliario, etc."; de modo que los espacios se organizan con estructuras ligeras que permiten la movilidad y la transformación del espacio ante diferentes situaciones y formas de aprendizaje.

A esta condición efímera de la organización espacial, se suman las condiciones adaptables de iluminación y acústica "ligadas a criterios de sostenibilidad y ahorro energético".

Las condiciones espaciales del centro escolar se diseñan para captar la atención y ser elemento activo del proceso de aprendizaje

Tal y como afirma Hertzberger (2008), el espacio debería siempre articularse de manera que los lugares creados sean unidades espaciales de 
dimensiones apropiadas y justa medida de aislamiento, de modo que puedan acoger el tipo de relaciones entre usuarios para las que han sido diseñados. En este sentido, cómo se articula un espacio es un factor de diseño decisivo. Por ejemplo, esta condición determina si el espacio creado va a ser adecuado para la actividad de un gran y único grupo de usuarios o si va a ser conveniente para su uso por parte de varios grupos, diferentes y más reducidos. Cuanto más articulado sea el espacio, más pequeña es la unidad espacial. Y cuantos más focos de atención haya, más actividades pueden ejercerse al mismo tiempo por diferentes grupos de usuarios.

Son varios los elementos que posibilitan la construcción de un espacio articulado y equilibrado: los desniveles, los focos de atención, las dobles alturas y las vistas cruzadas, el control de la luz, el control de la acústica del espacio, la disposición de materiales diversos "por ejemplo en el plano del suelo, creando un mapa de situaciones", etc. Todos ellos influyen en el modo en que un espacio se utiliza.

La aplicación de la crítica poética a la arquitectura de centros escolares lleva a la siguiente conclusión general: existe una correspondencia entre el modelo educativo y la arquitectura escolar que lo acoge. Y la interacción entre campo educativo y arquitectura se produce de modo bidireccional. De modo que, tanto el desarrollo de un modelo educativo concreto precisa de un espacio escolar acorde a él, como que la construcción de una arquitectura escolar concreta tiene como resultado la promoción de estímulos diversos para el aprendizaje. Lo que quiere decir que es posible establecer, para un espacio arquitectónico, criterios de diseño reactivos del modelo pedagógico correspondiente. Y, llevado al caso de la EDS, que es posible apuntar una serie de cualidades para la realidad del espacio escolar que, por sí mismas, van a acompañar positivamente el desarrollo de pedagogías acordes con los valores y criterios de la EDS.

\section{Discusión}

Los resultados que este trabajo aporta establecen que una interacción cuidada entre educación y arquitectura lleva consigo la construcción de centros escolares con espacios que propician el desarrollo de modelos educativos innovadores y avanzados. Espacios acordes con una EDS en los que sean condiciones principales la estimulación del estudiante - la estimulación de su creatividad, de su pensamiento crítico, de su autonomía, de sus capacidades cognitivas y emocionales-, las acciones participativas y colectivas "en equilibrio con los momentos intrapersonales", la educación en valores de sostenibilidad, etc.

Se confirma, así, que es posible establecer una relación de correspondencia entre el modelo pedagógico que se desarrolla en un espacio educativo y su cualidad arquitectónica. En este sentido, se ha logrado el objetivo de identificar criterios de diseño para un espacio arquitectónico que active modelos pedagógicos afines a la EDS. No obstante, la fortaleza de estas conclusiones es, a su vez, la limitación del trabajo. Los valores y criterios de la EDS hablan de sostenibilidad, creatividad, diversidad, interacción, participación, etc. Se trata de valores que, según los resultados obtenidos en este trabajo, se traducen directamente en espacios de diseño flexible, versátil y multifuncional "de la manera que se describe en los criterios del apartado anterior". Sin embargo, no es posible concretar condiciones normativas para el espacio. Los criterios de diseño, precisamente porque así lo es también el modelo educativo con el que busca su correspondencia, son necesariamente abiertos y flexibles. No condicionan el espacio si no es, precisamente, para establecer un espacio sin condiciones previas. En el sentido de evitar cualquier estructura que lo rigidice.

En cuanto a la discusión que este trabajo puede despertar con otros estudios y teorías fuente, se piensa que esta puede venir asociada a las teorías de la sociología del espacio o sociología de 
los atributos espaciales (Leal, 1997). La posibilidad de establecer una serie de criterios de diseño para la construcción de espacios educativos acompañantes de un modelo pedagógico concreto "en este caso afín a la EDS" conlleva la aceptación de este espacio como una realidad absoluta, incluso anterior a la dimensión espacio temporal que esta teoría presenta como condición necesaria. Esta posibilidad de establecer una serie de criterios de diseño para la construcción de espacios educativos establece una correspondencia entre la realidad física del espacio y una acción educativa concreta. El espacio precisa del usuario para activarse y, por tanto, necesita ser percibido; sin embargo, este trabajo establece que este mismo espacio es el elemento que activa una acción concreta por parte de los usuarios. El espacio es usado como quiere ser usado, se diseña para ser usado según unos criterios pedagógicos concretos. Su papel es, por tanto, de elemento reactivo. Influye de modo consciente en la percepción del usuario. En este sentido, los resultados presentan afinidad con las teorías de la neuroeducación (Mora, 2010).
En todo caso, los resultados obtenidos muestran que en los nuevos espacios educativos, afines a una educación para el desarrollo sostenible, tanto alumnos como docentes entienden el espacio desde nuevas potencialidades que procuran nuevas interacciones. Esto apunta a la necesidad de revisar la normativa estatal existente para el diseño arquitectónico de centros escolares públicos. El aprendizaje no se produce de forma lineal y, por lo tanto, la misma complejidad y riqueza se ha de contemplar en el espacio educativo, de cara a apoyar el aprendizaje y la comprensión. Se trata de convertir el espacio escolar —el centro escolar, su arquitectura- en un lugar para la experimentación, la percepción, la exploración y la relación no jerárquica; ya que el medio arquitectónico no solo induce funciones, facilitando o dificultando movimientos, promoviendo o entorpeciendo la ejecución eficaz de las tareas, etc., sino que, además, se hace lugar y educa mediante la transmisión de valores, promoviendo la identidad personal y colectiva, y favoreciendo ciertas formas de relación y convivencia (Romañá, 2004).

\section{Notas}

${ }^{1}$ En 2012, la UNESCO publica un reporte de seguimiento y evaluación del DEDS para su implementación.

${ }^{2}$ Estos objetivos se han renovado en la nueva Agenda de Desarrollo 2030 aprobada por la Organización de Naciones Unidas el 25 de septiembre de 2015, en la que garantizar una educación inclusiva, equitativa y de calidad; así como promover oportunidades de aprendizaje durante toda la vida para todos es el cuarto Objetivo de Desarrollo Sostenible (Organización de Naciones Unidas, 2015).

${ }^{3}$ En este sentido, hay que tener en cuenta que la arquitectura escolar puede contribuir a la EDS, no solo por la disposición del espacio, que acompaña un determinado modelo educativo, sino también por su incidencia directa en la sensibilización de los estudiantes y de la comunidad educativa en criterios de sostenibilidad ambiental; a través de otros factores, como la eficiencia energética de los edificios y la gestión coherente con principios y valores del desarrollo sostenible: por ejemplo, propiciar el reciclaje, el tratamiento de residuos, la movilidad sostenible, etc.

${ }^{4}$ A estos efectos, consultar el documento de Liliana Moraewitz (2014) que repasa los objetivos de la EDS como modelo pedagógico.

${ }^{5}$ Planos técnicos de los proyectos, memorias descriptivas y constructivas, e imágenes.

${ }^{6}$ En las tablas de resultados, se identifican del siguiente modo: caso A - Fuji Kindergarten; caso B - Ramat Chen Arts \& Science Elementary School; caso C - Vittra School Brotorp.

${ }^{7}$ Fuente espacio arquitectónico: Tezuka Architects: http://www.tezuka-arch.com/english/

${ }^{8}$ Fuente espacio arquitectónico: Studio Sarit Shani Hay: http://shanihay.com/

${ }^{9}$ Fuente espacio arquitectónico: Studio Rosan Bosch: http://www.rosanbosch.com/ .

${ }^{10}$ En este sentido, se toman como referencia el conjunto de tablas presentadas para los criterios de interacción binaria. 


\section{Referencias bibliográficas}

Altomonte, S., Rutherford, P., y Wilson, R. (2012). Mapping the Way Forward: Education for Sustainability in Architecture and Urban Design. Corporate Social Responsibility and Environment Management, Special Issue: The Influence of Pedagogy and Curriculum in Sustainable Development Education for Universities, Volumen 21, 3, 143-154. doi: 10.1002/csr.1311

Amann (2015). La crítica poética como instrumento del proyecto arquitectónico. Buenos Aires, AR: Diseño, 2015.

Aznar, A., y Ulls, A. (2009). La formación de competencias básicas para el desarrollo sostenible: el papel de la Universidad. Revista de educación, Número Extra 1, 219-237.

Burke, C., y Grosvenor, I. (2003). The School I'd Like: Children and Young People's Reflections on an Education for the $21^{\text {st }}$ Century. London, UK: Routledge.

Cabanellas, I., y Eslava, C. (2005). Territorios de la infancia. Barcelona, ES: Graó.

Casqueiro, F. (2014). Canon de centros escolares del siglo XX. Madrid, ES: Mairea Libros.

Castells, M. (1996). The Information Age: Economy, Society and Culture. Volumen I. The Rise of the Network Society. Cambridge, Massachusetts, USA: Blackwell Publishers.

Castells, M. (2009). Comunicación y poder. Madrid, ES: Alianza Editorial.

Ceppi, G., y Zini, M. (eds.) (1998). Children, Spaces, Relations: Metaproject for an Environment for Young Children. Milano, IT: Reggio Children / Domus Academy.

Cole, L. B. (2014). The Teaching Green School Building: a Framework for Linking Architecture and Environmental Education. Environmental Education Research, Volumen 20, 6, 836-857, doi: $10.1080 / 13504622.2013 .833586$

Dudek, M. (1996). Kindergarten Architecture: Space for the Imagination. London, UK: E \& FN Spon.

Dudek, M. (2000). Architecture of Schools: The New Learning Environment. Oxford, UK: Architectural Press.

Dudek, M. (2007). Schools and Kindergartens. A Design Manual. Basel, SW: Birkhauser Verlag.

Earth Charter International (2000). La Carta de la Tierra. Recuperado de: http://www.earthcharterinaction.org/invent/images/uploads/echarter_spanish.pdf

Gehl, J. (2011). Life Between Buildings: Using Public Space. London, UK: Island Press.

Geli, A. Ma., Junyent, M., y Sánchez, S. (eds.) (2004). Ambientalización curricular de los estudios superiores. Tomo III. Diagnóstico de la Ambientalización curricular de los estudios superiores. Girona, ES: Universidad de Girona, Publicaciones de la Red-ACES.

Giddens, A. (1991). Modernity and Self Identity. Cambridge, UK: Polity.

Goffman, E. (1963). Behaviour in Public Places: Notes on the Social Organizations of Gatherings. New York, USA: The Free Press.

Gottdiener, M. (1994). The New Urban Sociology. New York, USA: McGraw Hill.

Harvey, D. (1989). The Urban Experience. Oxford, UK: Basil Blackwell.

Harwood, E. (2010). England's Schools: History, Architecture \& Adaptation. London, UK: English Heritage.

Hertzberger, H. (2008). Space and Learning. Rotterdam, NL: 010 Publishers.

Kern, S. (1983). The Culture of Time and Space 1880-1918. Cambridge, Massachusetts, USA: Harvard University Press.

Kotnik, J. (2011). Guarderías: manual práctico y 37 proyectos. Barcelona, ES: Links Books.

Krokfors, L., Kangas, M., Kopisto, K., Rikabi-Sukkari, L., Salo, L., y Vesterinen, O. (2015). Learning. Creatively. Together. Educational Change Report 2016. Helsinki (FI): University of Helsinki.

Larrañaga, A. (2012). El modelo educativo tradicional frente a las nuevas estrategias de aprendizaje. Bilbao (ES): Universidad Internacional de La Rioja UNIR. 
Leal, J. (1997). Sociología del espacio: el orden espacial de las relaciones sociales. Política y Sociedad, 25, 21-36.

Lefebvre, H. (1976). La production de l'space. Paris, FR: Anthropos.

Max Neef, M. A., Elizalde, A., y Hopenhayn, M. (1986). Teoría del Desarrollo a Escala Humana. Santiago de Chile, CL: Upsala, CEPAUR.

Max Neef, M. A. (2006). Desarrollo a escala humana. Conceptos, aplicaciones y algunas reflexiones, con la colaboración de A. Elizalde y M. Hopenhayn. Barcelona, ES: Icaria.

Minguet, J. M. (2007). Arquitectura de guarderías-jardines de infancia y colegios. Sant Adrià de Besòs, ES: Instituto Monsa de Ediciones.

Miranda, A. (1999). Ni robot ni bufón. Manual para la crítica de arquitectura. Madrid, ES: Cátedra.

Moles, A., y Rohmer, E. (1998). Psychologie de l'espace. Paris, FR: L'Harmattan.

Mora, F. (2010). Neuroeducación: solo se puede aprender aquello que se ama. Madrid, ES: Alianza Editorial.

Morawietz, L. (2014). Educación para el Desarrollo Sostenible y el Cambio Climático. Apuntes. Educación y Desarrollo Post-2015, 2.

Morin, E. (1980). Le Méthode II: La vie de la Vie. París, FR: Editions du Seuil.

Muntañola, J. (2004). Arquitectura, educación y dialogía social. Revista Española de Pedagogía, 228, 221-228.

Nedved, M., y Zámecníková, V. (2014). Influence of Alternative Education on the Architecture of Conventional Schools. Advanced Materials Research, 1020, 686-691.

Organización de Naciones Unidas (1987). Brundtland, G. (Presidente de la Comisión). Nuestro futuro común. Asamblea General de las Naciones Unidas, Informe A/42/427. Recuperado de: http://www.un.org/es/comun/docs/?symbol=A/42/427

Organización de Naciones Unidas (2002). Decenio de las Naciones Unidas de la Educación para el Desarrollo Sostenible 2005-2014. Resolución $n^{o}$ 57/254 de la Asamblea General de las Naciones Unidas.

Organización de Naciones Unidas (2015). Proyecto de documento final de la cumbre de las Naciones Unidas para la aprobación de la agenda para el desarrollo después de 2015. Asamblea General, Seguimiento de los resultados de la Cumbre del Milenio. Recuperado de: http://www. un.org/es/comun/docs/?symbol=A/69/L.85

Perkins, B., y Kliment, S. A. (2001). Building Type Basics: Elementary and Secondary Schools. New York, USA: John Wiley \& Sons Inc.

Romañá, T. (2004). Arquitectura y educación: perspectivas y dimensiones. Revista Española de Pedagogía, 228, 199-220.

School Buildings and Design Unit, Departament for Education and Skills (UK) (2003). Classrooms of the Future: Innovative Designs for Schools. London, UK: The Stationary Office.

Sennett, R. (1996). Flesh and Stone: The Body and the City in Western Civilization. New York (USA): W. W. Norton \& Company.

Simmel, G. (1908). Soziologie. Untersuchungen über dir Formen der Vergesellschaftung. Leipzig, DE: Duncker \& Humblot.

Tornaghi, Ch., y Knierbein, S. (Eds.) (2014). Public Space and Relational Perspectives: New Challenges for Architecture and Planning. London, UK: Routledge.

UNESCO (2005). Links Between the Global Initiatives in Education. "Education for Sustainable Development in Action", Technical Paper 1.

UNESCO (2012). Shaping the Education of Tomorrow: 2012. Report on the UN Decade of Education for Sustainable Development, Abridged. Paris, FR: United Nations Educational, Scientific and Cultural Organization. Recuperado de http://unesdoc.unesco.org/images/0021/002191/219155s.pdf 
UNESCO (2014). UNESCO Education Strategy 2014-2021. Paris, FR: United Nations Educational, Scientific and Cultural Organization. Recuperado de http://unesdoc.unesco.org/ images/0023/002312/231288e.pdf

Verstegen, T. (ed.) (2008). Contemporary Dutch School Architecture: A Tradition of Change. Rotterdam, NL: Nai Publishers.

Wright, S., y Beard, A. (eds.) (2006). Century 21 Schools. Birmingham, UK: Imaginative Minds.

\begin{abstract}
The relationship between architecture and Education for Sustainable Development (ESD). Space as a reactive factor within the pedagogical model
\end{abstract}

INTRODUCTION. Education for Sustainable Development (ESD) promotes participatory pedagogical models. Its principal conditions are to promote student motivation and autonomy through the acquisition of critical thinking, based on values of sustainability. This means the introduction of changes in current teaching methods. Does it also mean the need for a different type of school architecture? Can an architectural space positively accompany the ESD? Therefore, the objectives that guide this paper are: to answer these questions and identify a potential design criteria for an architectural space accompanying the pedagogical model. METHOD. The study uses an inductive methodology that works with the poetic criticism of architecture (a specific analysis tool), which analyzes the interactions between systems that build a space. Thus, one can identify relationships of convergence and divergence between educational models and the spatial environment. Three international schools are taken as reference. These represent examples of educational models which include the values and criteria of ESD. These schools have been built during the 2005-2014 decade, a period that marks the objectives of UNESCO for the development of ESD. RESULTS. The paper shows that there is an interaction between the pedagogical model and educational space. Futhermore, as the main finding, twelve design criteria - ten specific and two general — are identified for the school environment to accompany ESD pedagogical models. DISCUSSION. On one hand, the results show affinity to theories in the emerging science of Neuroeducation. On the other hand, the study finds its limit in the achievement of its objectives; because the criteria identified are open criteria. These results point to the need to renew the existing national regulations for architectural public schools, so that the implementation of innovative pedagogical models are possible.

Keywords: School buildings, Physical environment, School epace, Design requirements, Open education.

\title{
Résumé
}

L'Éducation pour le Développement Durable (EDD) et architecture scolaire. L'espace comme réactif dans les modèles pédagogiques

INTRODUCTION. L'Éducation pour le Développement Durable (EDD) favorise des modèles pédagogiques participatifs, dans lesquels se développent la motivation et l'autonomie des élèves grâce à l'acquisition de la pensée critique fondée sur les valeurs du développement durable. Tout

162 - Bordón 68 (I), 2016, 145-163, ISSN: 0210-5934, e-ISSN: 2340-6577 
cela signifie, entre autres modifications de base, l'introduction de changements dans les méthodes pédagogiques actuelles. Est-ce que cela signifie également la nécessité d'une architecture scolaire différente ? L'espace architectural peut-il accompagner positivement l'EDD? Lobjectif qui guide ce travail est celui d'identifier des critères de design potentiels pour un espace réactif du modèle pédagogique qui serait cohérent avec l'EDD. MÉTHODE. Létude utilise une méthodologie inductive qui fonctionne avec un outil d'analyse spécifique, la critique poétique de l'architecture, qui analyse les interactions entre les systèmes qui constituent un espace. Ainsi, on peut identifier des relations de convergence et divergence entre des modèles éducatifs et de qualité de l'espace. On a pris comme référence trois écoles internationales qui mettent en వuvre des modèles pédagogiques inclusifs des valeurs et critères de l'EDD. Ces écoles ont été construites au cours de la décennie 2005-2014, une période qui marque les objectifs de l'UNESCO pour le développement de l'EDD. RÉSULTATS. Larticle soutient qu'il existe une interaction entre le modèle pédagogique et l'espace éducatif. La découverte principale, identifie dix critères de design spécifiques et deux plus généraux pour que l'espace scolaire soit un espace d'accompagnement des modèles pédagogiques liés à l'EDD. DÉBAT. D'une part, les résultats montrent des affinités avec les théories de la Neuroéducation, comme une science émergente. D'autre part, l'étude trouve sa limite précisément dans la réalisation de ses objectifs ; en effet, les critères identifiés sont des postulats ouverts. Ces résultats soulignent la nécessité de renouveler la Réglementation de l'État existante concernant l'architecture des écoles publiques, de sorte qu'il soit rendu possible la mise en \uvre de modèles pédagogiques innovateurs.

Mots clés: Architecture scolaire, Environnement physique, Espace scolaire, Critères de design, Éducation ouverte.

\section{Perfil profesional de la autora}

\section{Beatriz Amann Vargas}

Arquitecta (UPV/EHU, 2002) y doctora en Arquitectura con la calificación de sobresaliente cum laude (Universidad Politécnica de Madrid, Departamento de Proyectos Arquitectónicos, 2014). Publica su tesis La crítica poética como instrumento del proyecto arquitectónico en la colección Textos de Arquitectura y Diseño (2015). Actualmente es la coordinadora académica de la Escuela de Design en el Istituto Europeo di Design Madrid.

Correo electrónico de contacto: b.amann@madrid.ied.es

Dirección para la correspondencia: calle Flor Alta, 8. C.P. 28004. Madrid. 
